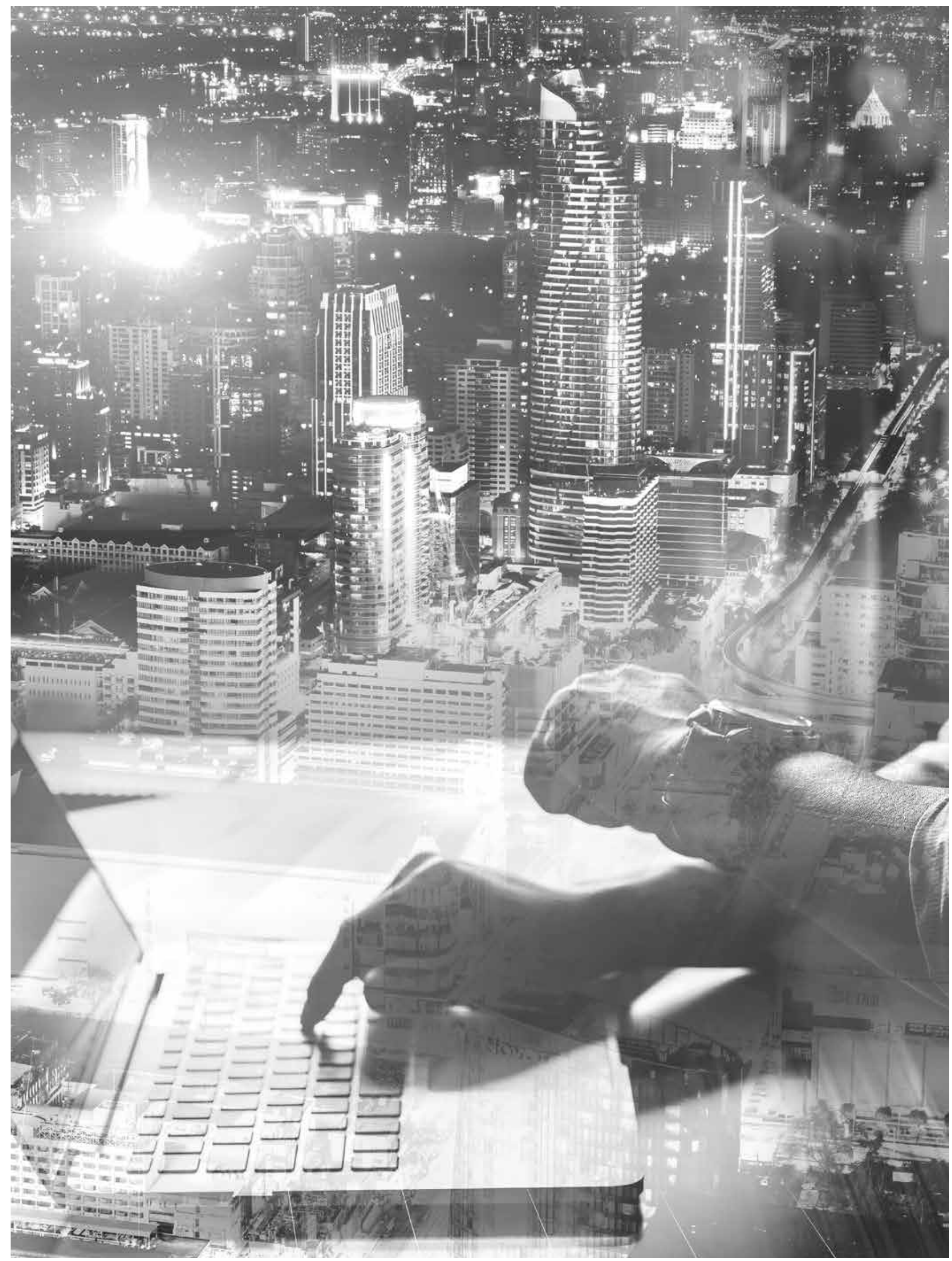




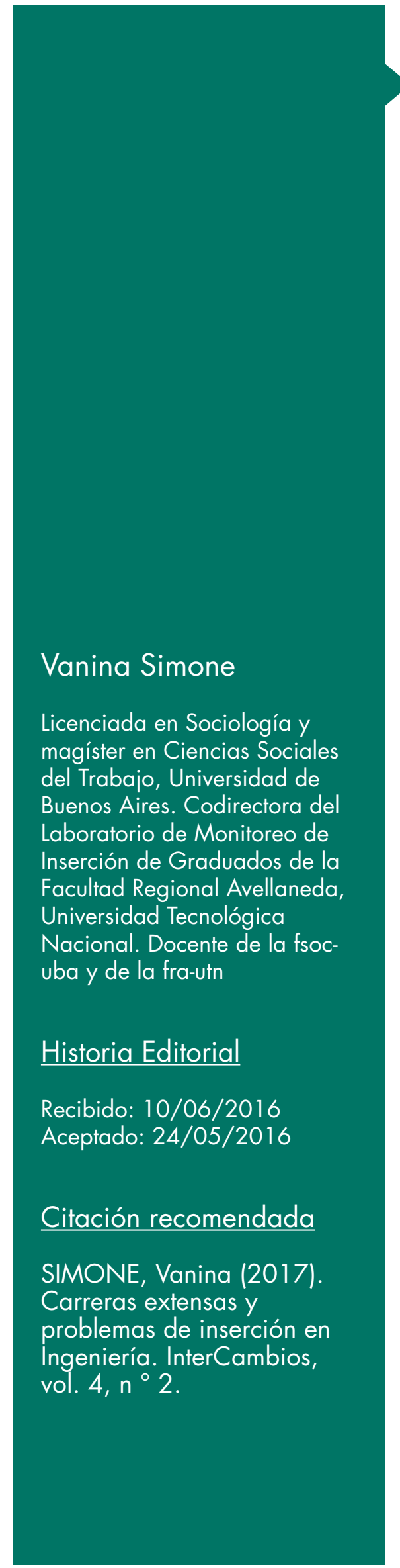

\section{Carreras extensas y problemas de inserción en Ingeniería}

\section{Extended careers and problems of insertion in labor market in Engineering}

Resumen

El artículo busca relacionar las situaciones de interrupciones y prolongación de los estudios universitarios con el desinterés por la obtención de la titulación en los plazos correspondientes y con las condiciones de empleo y las características de los sectores demandantes de los profesionales. Esta situación parece ir en sentido contrario de los requerimientos laborales de una profesión como la ingeniería civil, ya que la regulación requiere de un título habilitante debido a la responsabilidad civil que conlleva su actividad. El estudio de los relatos biográficos de los ingenieros civiles permite identificar tres factores que interrelacionados explican las trayectorias de formación con períodos de interrupciones, la duración de carrera más elevada que el promedio y los recorridos profesionales insatisfactorios. El primero es de carácter educativo-institucional, el segundo es de contexto económico y de mercado de trabajo, y ambos redundan en una inclinación hacia la desvalorización de la titulación. Esta última significa que la obtención del título no se traduce linealmente en logros significativos a nivel laboral y que el diploma no asegura una posición definida en el mercado de trabajo profesional.

Palabras claves:

Ingeniería, planes de estudio, demanda laboral, ejercicio de la profesión, expectativas postitulación.

\section{Abstract}

This article seeks to relate the interruptions and extension of the university studies with the lack of interest in obtaining the degree within the corresponding time, on the one hand, and, on the other, with the conditions of employment and the characteristics of the industry sectors where these professionals are demanded. The delay in obtaining the degree seems to go against the requests for a profession like civil engineering, since its practice requires a degree due to the liability it implies. The study of engineers' story lives allows to identify three interrelated factors that explain the educational paths with periods of interruptions, the extent of the careers longer than the average and unsatisfactory professional experiences. The first factor is of an educational and institutional character, while the second one relates to the economic context and the labor market. Both of them have an impact on the third factor: the depreciation of the qualifications. This last one means that assuring the obtainment of the degree is not reflected in significant achievements at work and the degree does not guarantee a defined position in the professional labor market.

Keywords:

Engineering, curriculum, labor demand, professional practice, post-degree expectations. 


\section{Introducción}

Las reflexiones de este artículo intentan relacionar las situaciones de interrupciones de los estudios universitarios y de desinterés por la obtención de la titulación en los plazos correspondientes con las condiciones de empleo y las características de los sectores demandantes de los profesionales. En este caso, los profesionales en estudio son los ingenieros en construcciones y los ingenieros civiles graduados en años recientes en la Universidad Tecnológica Nacional de Argentina.

Este trabajo se enmarca en la perspectiva de estudios longitudinales, que supone el seguimiento de las cohortes por el investigador desde su ingreso y a lo largo del tiempo (Landi y Giuliodori, 2001). Algunos trabajos en esta línea de las trayectorias educativas y laborales son los de Panaia (2006a) para el caso de los ingenieros tecnológicos, y los de Longo (2008) y Jacinto (2010) para el caso de jóvenes egresados del nivel secundario.

Al interés por tratar el problema de la graduación y el abandono, se agrega para las universidades argentinas la problemática de la extensión de la duración real de los estudios. Según indican las estadísticas de las universidades, el promedio es de ocho años, y según el análisis del sistema educativo universitario, la duración de los estudios se extiende por plazos que superan ampliamente el tiempo teórico de los planes de estudios (Landi y Giuliodori, 2001). Para el caso de las ingenierías, de acuerdo a los relevamientos de los laboratorios de monitoreo de inserción de graduados - en adelante laboratorios MIG-instalados en algunas facultades de Ingeniería de la Argentina ${ }^{1}$ y los que sintetiza la Secretaría de Políticas Universitarias del Ministerio de Educación, la duración real supera el promedio de ocho años para ubicarse en los 10 y 11 años. Los estudiantes de Ingeniería presentan importantes niveles de interrupción y abandono. Según Enrich (2014) y Simón (2015), situaciones similares acontecen en las carreras de Ingeniería en la Universidad de la República en Uruguay. Así como el denominado «abandono tardío» relacionado con la dificultad de compatibilizar las actividades laborales cada vez más demandantes con la terminación de la formación.

El abordaje de estas problemáticas a partir de un marco teórico-metodológico longitudinal y procesual permite evitar la estigmatización e individualización de quien que no ha concluido la formación o la ha abandonado temporalmente y asimismo comprender al estudiante como sujeto de cambio permanente, con capacidades de decisión y posibilidades de aprendizaje que se renuevan. De este modo, el análisis de las trayectorias no se realiza de acuerdo al supuesto de un proceso lineal con etapas consecutivas (etapa educativa para luego ingresar a la etapa laboral), sino de un proceso que puede ser fragmentado, que implica discontinuidades y cambios en las prioridades del proyecto de vida. Se trata, entonces, de analizar las diversas historias - familiares, educativas, laborales y personalesque se conjugan e interrelacionan para comprender los cambios y las diferencias en los itinerarios profesionales, así como sus imbricaciones con los contextos económicos, sociopolíticos y del mercado de trabajo profesional. En función de este objetivo se torna central el uso de la técnica biográfica que pone el énfasis en la recuperación del sujeto a través de su historia. Esta técnica posibilita el análisis de la dimensión temporal como medio para comprender los procesos de cambio. Aquí se retoma la propuesta de Godard y Cabanés (1996) de temporalidades sociales; como explica Panaia (2006b), la idea es ir más allá de la centralidad de la subjetividad y de la estructura narra- tiva, para recuperar el ser sociológico. Esta comprensión se realiza sobre un relato argumentado que construye el sentido de un recorrido determinado, de encadenamiento de situaciones y decisiones. Como indican Cabanes y Godard (1996), cuando se recolectan los relatos biográficos, el problema es que se hace necesaria la comparación. Para ello los materiales tienen que ser suficientemente homogéneos, además de contar con un modelo de análisis que permita realizar posteriormente ese tipo de ejercicio. El modelo que proponen estos autores es el procesual o modelo del itinerario, que trata de comprender cómo se estructuran los procesos de encadenamiento a lo largo de una vida (Cabanes y Godard, 1996: 26). En este esquema se explica cómo o hasta qué punto el itinerario biográfico se modifica en el transcurso o desarrollo temporal. Sin embargo, la reconstrucción que realiza el sujeto de su propio recorrido no deja de ser parcial, con elecciones sobre qué ocultar y qué resaltar, evocación de los tipos de recuerdos, entre otros. Los relatos de vida son producciones subjetivas inseparables de los actos interpretativos (Pailot, 2003). Como sugiere Bertaux (citado en Pailot, 2003), las historias de vida más que como historias de una "vida» tendrían que considerarse relatos de las prácticas.

En función de este enfoque se analizan 23 entrevistas biográficas de ingenieros civiles y en construcciones de las cohortes 2006, 2007 y 2008 de la Facultad Regional Avellaneda de la Universidad Tecnológica Nacional (en adelante UTN). La UTN es la única universidad de Argentina que tiene a las ingenierías como objetivo prioritario. Esta institución pública presenta un carácter federal con 29 facultades en todas las regiones del país y en ella cursan el $50 \%$ de los estudiantes de Ingeniería del país. Cabe aclarar que este trabajo parte de la investigación cuantitativa y cualitativa que lleva a cabo el Laboratorio MIG de la fa-

1 Según el Laboratorio mig de la Facultad Regional Pacheco de la UTN, para los ingenieros civiles graduados entre los años 2000 y 2004 la duración promedio de la carrera es de 11 años (Formento y otros, 2007). 
cultad sobre los graduados de las cohortes 2006, 2007, 2008 y 2009 de las seis especialidades de ingeniería que se dictan, y posibilita un análisis comparativo de sus comportamientos y características según las diferentes carreras y sus campos de actividad profesional (Simone y Wejchenberg, 2013). Si bien la combinación de estudio y trabajo es característica de la mayoría de los estudiantes y graduados de Ingeniería de la UTN, y por ello se explica —en parte- la prolongación de los estudios, los resultados muestran en los ingenieros civiles situaciones de interrupciones prolongadas, dificultades para lograr la titulación y el paso por proyectos institucionales de apoyo a la graduación. Estos hallazgos parecen ir en sentido contrario a los requerimientos específicos de esta especialidad, ya que la regulación requiere para su ejercicio el título habilitante y la matriculación en colegios profesionales, debido a la responsabilidad civil que conlleva su actividad. En vista de ello, en este trabajo se decide profundizar en la comprensión de las trayectorias de los graduados de esta especialidad. El estudio de los relatos biográficos permite identificar tres factores que interrelacionados echan luz sobre las trayectorias de formación con períodos de interrupciones, con una duración de carrera más elevada que el promedio y con recorridos profesionales insatisfactorios en virtud de las expectativas y las incumbencias del rol. El primer factor es de carácter educativo-institucional, el segundo es de contexto económico-sectorial y de mercado de trabajo, y ambos redundan en una inclinación hacia la desvalorización de la titulación. Esta última significa que, en la mayoría de los casos estudiados, con amplia expe- riencia laboral en el rubro, la obtención del título no se vivencia como un recurso significativo para afrontar los desafios del ámbito laboral, ya que el hecho de poseerlo no garantiza linealmente una posición definida en el mercado de trabajo profesional para quienes lo tienen. ${ }^{2}$ Se observan dificultades como una desdibujada delimitación del rol, bajo nivel de salarios, imposibilidad de acceder a cargos jerárquicos, malas condiciones de empleo y subordinación respecto de otros profesionales. A continuación se desarrollan los tres aspectos.

\section{La incidencia del cambio de plan} y el régimen de estudio

Los antecedentes de la carrera de Ingeniería Civil se remontan al año 1955, a la creación de la Facultad Regional Avellaneda en el marco de la anterior Universidad Obrera Nacional. Allí se dictaban las carreras de Ingeniería en Construcciones de Edificios y de Construcciones de Obra, ambas de seis años de duración. La instrucción impartida en la universidad se orientaba a formar ingenieros con espíritu práctico, condiciones ejecutivas y habilidad manual; sus planes profundizaban la especialización, es decir, la profundización del conocimiento en un área determinada. A ello se adicionaba la obligación para los alumnos de trabajar en la industria en una rama afín a los estudios cursados. Esta característica aseguraba el proceso de transmisión de los conocimientos teóricos a la práctica del taller o la planta industrial y determinaba el horario vespertino de las clases. Luego de algunos cambios menores y ya con la nueva denominación de Universidad
Tecnológica Nacional, se produce un nuevo cambio en la titulación en el plan de 1975, pasando a denominarse Ingeniería en Construcciones. En los planes siguientes, 1979, 1985 y 1988, la especialidad conservó esa denominación. La modificación importante llegó con el Plan 1988, que permitió obtener, además, el título en Ingeniería Civil realizando un complemento de asignaturas en sexto año. En este plan ingresaron la mitad de los graduados analizados en este trabajo. Sin embargo, la mayor reforma en esta especialidad se observó hacia mediados de la década del 90. En primer lugar, con el Plan 1995 el título de Ingeniería Civil deja de dictarse como complemento y pasa a ser el único título de la especialidad. En segundo lugar, la duración del plan de estudios pasa de seis años - $\mathrm{O}$ siete en total, en caso de continuar con el complemento de Civil- a cinco años y un semestre.

El cambio en la titulación que introduce el Plan 1995 marca la diferenciación establecida por el Decreto Nacional 256/94 entre los alcances del título (las actividades para las que resulta competente un profesional en función del perfil del título y de los contenidos curriculares de la carrera) y las incumbencias (aquellas actividades comprendidas en los alcances del título cuyo ejercicio pudiese comprometer el interés público). Para este caso, el nuevo plan abrió un debate acerca de las incumbencias que tendría el graduado en Ingeniería Civil de la UTN.Ya que además de los alcances del título y las incumbencias del ingeniero en construcciones, el ingeniero civil puede desempeñarse en construcciones de vialidad e hidráulica, que requieren conocimientos de cálculo estructural. Las nuevas pautas

2 Esta situación da cuenta de un mercado profesional en disputa y una colegiación que parece tener dificultades para delimitar el campo ocupacional propio a partir de las incumbencias que otorga el título. En el sentido de pensar a las profesiones como formas históricas de coalición de actores que defienden sus intereses tratando de asegurar y mantener un cierre de su mercado de trabajo (Panaia, 2004). No se trata de una crítica a la función social del título, sino de un aporte a la reflexión sobre los distintos factores que desalientan el logro del diploma en los tiempos asignados por los planes de estudios. Además, la categoría de ingeniero significa en primer lugar un título académico, pero además se reconoce en la empresa con ese nombre una función profesional. No obstante, como continúa Panaia, no todos los que tienen el título ejercen esa función y muchos que no lo tienen la ejercen (Panaia, 2004: 196). Tampoco es una categoría comparable entre distintos países, ya que son diferentes los contenidos que se le dan, la duración de los estudios y el estatuto profesional que rige para el ejercicio de la profesión (Panaia, 2004: 196). 
del diseño curricular sostienen que el espacio de electivas permite una formación que acompaña la concepción de la ingeniería civil como «actividad amplia», favoreciendo la flexibilización del campo de trabajo del egresado (Ordenanza 1030) (Simone y otros, 2007).

Según el perfil actual, el ingeniero civil se encarga de resolver los problemas de infraestructura para la producción de bienes y servicios del país en general: edificios, fábricas, viviendas, puentes, carreteras, vías ferroviarias y navegables, puertos y aeropuertos, aprovechamientos hidroeléctricos, sistemas de riego, defensas aluvionales, distribución de agua, desagües pluviales, cloacas, industriales. También entiende de seguridad, mantenimiento y operación, modernización, planificación, control ecológico y eficiente reemplazo de la infraestructura teniendo en cuenta los aspectos técnico-económicos.

Desde la puesta en marcha del Plan 1995 - que con leves modificaciones es el que continúa hasta hoy- en la carrera de Ingeniería Civil se graduaron 179 estudiantes en el período de 19 años que va de 1995 a 2013, con un promedio anual de nueve casos. El promedio total -incluyendo las seis especialidades - es de 77 graduados por año. Las tres cohortes de graduados, 2006, 2007 y 2008, con titulaciones de la especialidad suman 42 casos. Si bien los casos entrevistados representan más de la mitad de los ingenieros graduados en la especialidad en la UTN-FRA, los resultados de este análisis deben inscribirse como casos ilustrativos de los comportamientos de este grupo profesional, tanto respecto a la extensión de sus carreras universitarias como a las dificultades en el ejercicio profesional.

A partir de su análisis, se detectan casos que ingresaron en etapas anteriores a 1995 y por consiguiente en planes de estudio que no rigen en la actualidad. Se trata de trayectorias con cursadas completas pero que abandonan la formación con una cantidad numerosa de finales pendientes de rendir y luego retoman los estudios y aprueban los exámenes finales en el marco de un proyecto institucional especial de apoyo a la graduación. El régimen de cursada en la FRA es anual y en el Plan 1988 todas las materias tienen final obligatorio pero sin límite temporal de aprobación, es decir que la regularidad de las materias no tenía vencimiento. Ante la considerable cantidad de estudiantes con cursadas completas pero con exámenes finales pendientes y desvinculados de la institución, ${ }^{3}$ en el año 1998 la facultad puso en marcha un proyecto específico que apuntaba a promover la titulación y convocar a aquellos estudiantes que no completaron la carrera. Se denominó Proyecto Alfa y consistió en un sistema de tutorías, clases y mesas de finales especiales con el fin de volver a vincular a los estudiantes con la carrera y conformar grupos de estudio que compartían condiciones similares. Además, y luego del egreso, en algunos casos se los convocó para ser tutores de los primeros años, ayudantes de cátedra o investigadores en laboratorios. ${ }^{4}$ Con el apoyo del Proyecto Alfa se graduaron 180 nuevos profesionales (Arana, 2004) entre los años 1998 y 2002. En ese contexto, ante las recurrentes situaciones de abandono con finales pendientes y extensión de la duración de la carrera una vez finalizada la cursada - años solamente de rendición de finales-, a partir del año 2000 se cambió el régimen de estudio y la regularidad de la materia vence a los cuatro años.

Ante la falta de límite en el vencimiento de las materias en el Plan 1988, aquellos que cursaron en dicho plan coinciden en que esta falta de regulación se transforma en uno de los factores que desmotivan la finalización de la carrera, a pesar de que, como se ha mencionado, el título es una exigencia para el ejercicio profesional. El Proyecto Alfa logró en esos casos recomponer esas trayectorias interrumpidas e integrar a la comunidad educativa y a la especialidad los valiosos aportes cognitivos, sociales e individuales de los estudiantes que la misma institución ayudó a formar y crear. El cambio de plan en el año 1995 también impactó en las trayectorias de quienes ingresan en una carrera y culminan en otra, con modificaciones además en las incumbencias. En la heterogeneidad de situaciones, las consecuencias de los momentos de transición en el sistema educativo se ven claramente en las trayectorias individuales de aquellos que atravesaron los cambios. Como plantea $\mathrm{Pa}-$ naia (2013) citando a Eckert (2005), las conductas de interrupción, si bien son individuales, afectan las trayectorias de los grupos sociales por el solo hecho de su ocurrencia, y hacen que estos se vean más vulnerables a la aparición de este tipo de conductas. En este sentido, aquellos graduados con interrupciones y prolongación de las carreras se reconocen como «de otra época» en la cual la no titulación no era significativa para las carreras laborales y la interrupción formaba parte

3 Esta situación se observa en otras facultades de la uTN, como la de General Pacheco, en la zona norte del Gran Buenos Aires. Como describe Panaia (2006a), entre aquellos que cursaron con planes anteriores al año 1995, se detecta una población que teniendo todas las materias cursadas no rindió sus finales. El permitir el reglamento la no exigencia de rendición de finales para inscribirse en las materias correlativas permitió una cursada rápida pero con poca rendición de finales, y su acumulación hizo casi imposible para estos estudiantes lograr el título (Panaia, 2006a: 304).

4 Una experiencia similar es descrita en el año 2011 por el Programa de Respaldo al Aprendizaje de la Facultad de Veterinaria de la Universidad de la República de Uruguay respecto a la experiencia de ofrecer apoyo y acompañamiento a estudiantes para apuntalar el egreso. Esta experiencia implica generar movimientos hacia el interior de la universidad y generar estrategias que se traducen en «ir hacia el estudiante», promoviendo de esta forma la identificación de las redes de apoyo con las que cuentan para construir y reconstruir sus procesos de aprendizaje y preparase así para el egreso (Lujambio y otros, 2013). 
de situaciones «naturalizadas» para estos grupos. A estas situaciones se agrega el cambio de plan y de título que para estos grupos implica decidir si cambiarse al nuevo plan o graduarse en el plan anterior o de origen. De todas formas, los casos analizados que ingresaron en años anteriores al cambio de plan quedaron en su mayoría identificados con el título de Construcciones y sus elecciones laborales y vocacionales se diferencian de los perfiles que asumen quienes eligen la carrera de Ingeniería Civil en el nuevo plan. Los acontecimientos de índole educativo-institucional descritos inciden en las trayectorias y marcan diferencias a partir de las cuales se pueden discriminar dos subgrupos dentro de esta población compuesta por las tres cohortes con predominio de varones. ${ }^{5}$ Ambos se conforman por una cantidad equivalente de casos, es decir que el total se divide en dos. El primero agrupa a los ingenieros que ingresan a la carrera en años anteriores al cambio de plan (1995) y el segundo a quienes ingresan con el nuevo plan.

En el primer grupo predominan los maestros mayores de obra, a quienes esta situación les permite incorporarse a la vida laboral mediante el ejercicio de dicha ocupación con o sin la matriculación correspondiente y en la modalidad independiente. Al principio son pequeños trabajos «de juventud» que combinan con la producción de planos y diseños. Esta modalidad laboral independiente va marcando un camino a lo largo de la trayectoria; la complejidad de las tareas va aumentando y diversificándose a medida que por un lado se consiguen clientes y contactos, y que por otro avanzan en los estudios de grado. Esta condición laboral a veces es acompañada por la docencia en escuelas medias y en la UTN. La combinación del empleo independiente como profesionales por cuenta propia y la docencia en las escuelas técnicas medias se mantiene hasta el momento en que son entrevistados - a tres años de la graduación-.

En cambio, los graduados que ingresaron a la universidad hacia mediados de la década del 90 provienen de escuelas técnicas y no técnicas, han obtenido bachilleratos, títulos comerciales y son maestros mayores de obra. El primer contacto con el mercado laboral lo hacen en la modalidad de relación de dependencia, en sectores relacionados con lo ingenieril y la construcción; el crecimiento también es paulatino y se construye a través de la experiencia laboral y los conocimientos que se van adquiriendo en la facultad y en el trabajo. Se podría trazar un recorrido típico: dibujantes, cadistas, proyectistas, calculistas, hasta puestos de dirección con supervisión de proyectos que se suelen obtener cuando se está por concluir los estudios universitarios o una vez obtenido el título (Simone, Iavorski y Somma, 2013).

En el punto siguiente se resumen algunas consideraciones del contexto económico y sectorial de la construcción como principal sector demandante de estos profesionales. Se describen sus principales características y su conformación, ya que es el segundo factor — según el análisis- que imprime su marca en las trayectorias de los graduados.

\section{La actividad de la construcción y} el mercado de trabajo

La actividad de la construcción es muy sensible a los ciclos de la economía y a las políticas económicas de ampliación o de contracción del mercado interno y al comportamiento de la inversión pública. Además, presenta características estructurales que la di- ferencian del resto de los sectores de la economía. Según Coremberg (2000), ellas son: a) alta heterogeneidad de los productos y consiguiente reducida estandardización en la producción; b) dispersión de la localización geográfica de la demanda; c) convivencia de perfiles productivos disímiles; d) mercados de trabajo duales como consecuencia del uso de tecnologías tanto tradicionales como intensivas en el empleo de mano de obra calificada; e) alta concentración empresaria a nivel de grandes obras junto con una amplia rotación de empresas pymes que realizan obras de menor escala y empresas subcontratistas especializadas en determinados ítems de obra; f) stock de activos inmobiliarios. La vivienda es el activo que mayor ponderación tendría en el stock de capital y sobre todo en el stock de riqueza; por ello resulta de suma importancia el análisis de los efectos riqueza que produce la variación de precios de este tipo de activos, y la construcción es uno de sus principales determinantes (Coremberg, 2000: 2).

La apertura temporal en la que los ingenieros analizados estudian y trabajan abarca las décadas de los 80 , los 90 y la primera del siglo XXI. El contexto recesivo de la década de los 80 y la hiperinflación de los últimos años implicaron, como marca Coremberg (2000), que el nivel de actividad del sector durante ese período se mantuviera en niveles mínimos, principalmente asociado a la actividad especulativa en el mercado inmobiliario de alquiler de viviendas y oficinas. El retiro del Estado de las actividades productivas generó una mayor informalización de las relaciones laborales y una fuerte tendencia a realizar el proceso productivo mediante la subcontratación.

A partir de la estabilidad macroeconómica y las reformas estructurales de la década de los 90, la reducción

5 La ingeniería es una profesión culturalmente asociada a rasgos masculinos. Tanto en la matrícula de estudiantes como en la cantidad de graduados las mujeres son minoría y se concentran en aquellas especialidades de la ingeniería más vinculadas a la biología o a la administración. Los datos para las cohortes de graduados 2006 y 2007 para las seis carreras de ingeniería que se dictan en la FRA arrojan que solo un 7 \% pertenecen al sexo femenino. En la población de ingenieros de especialidad analizada en este trabajo solo un caso corresponde a una mujer con el título de construcciones. 
del racionamiento del crédito y el crecimiento del ingreso real permiten una recuperación de la demanda de obras de construcción. Estimaciones propias de la autora indican que la construcción de viviendas aumenta su ponderación en el total del valor bruto de producción de la construcción, llegando al 60 \% en 1997. Es la tipología de obra con mayor incidencia en el nivel total de la construcción (Coremberg, 2000).

Luego de la crisis económica y social de los años 2001 y 2002, comienza a evidenciarse una recuperación del sector que se mantiene fuertemente hasta 2006; a partir de allí se registra un marcado estancamiento. En este período el bloque que primero se reactiva es el de las viviendas, seguido de las obras de infraestructura y las obras viales. La proporción de inversión pública crece a partir del año 2006 y se destina a la construcción de viviendas; siguen en importancia las obras viales, luego las líneas de alta tensión, las obras ferroviarias y luego las de saneamiento (Ruggirello, 2011). Este predominio de las obras edilicias y de vivienda en el sector es consecuente con las áreas y rubros en los que se emplean la mayoría de los graduados en los años 80 y 90 ; recién después de 2003 se registran trabajos en obras de infraestructura, viales y de transporte y luego de saneamiento. Este paralelismo muestra los condicionamientos contextuales que moldean las trayectorias de los ingenieros al posibilitar pocos márgenes de maniobra para las decisiones de carrera profesional. En otras palabras, las decisiones sobre las propias carreras profesionales no podrían comprenderse sin tener en cuenta los marcos contextuales de la actividad laboral que delimitan — sin imposibilitar- las formas de ejercicio de la profesión y marcan horizontes posibles.

Este subsector de construcción edilicio habitacional es el más dinámico y con mayores niveles de estabilidad, y al mismo tiempo es el sector en el que tienen incumbencias los arquitectos. Este grupo profesional ocupa una posición de liderazgo en la gestión del diseño y la realización del producto. En este esquema, los ingenieros son convocados para realizar una parte del trabajo de diseño (planos, cálculos, reglamentaciones). Son principalmente los ingenieros titulados en construcciones quienes plantean esta tensión con los arquitectos, la falta de definición del rol del ingeniero en el subsector y por consiguiente la poca valoración de la obtención del título.

[...] siempre trabajé [haciendo cálculos] en estudios de arquitectura, porque el monopolio de la construcción lo tienen los estudios de arquitectura. (Federico, ingeniero en construcciones, abril 2012)

En los relatos, cuando ellos hacen referencia a sus expectativas respecto de la continuidad laboral en una misma empresa, hacen un análisis temporal de acuerdo a sus intereses, pero también incluyen una importante cuota de incertidumbre ligada a los ciclos de la economía. En las palabras del ingeniero civil entrevistado:

[...] Sí, dos años más para incursionar en la articulación entre "hacer papel" [hacer planos $y$ trabajos de la oficina técnica] y cómo se construye [la obra] [...]. - Y agrega-: todo depende de los vaivenes económicos, mi carrera en particular depende de si alguien invierte para construir. (Leandro, ingeniero civil, julio 2010)

En las épocas de crisis o recesión económica, el trabajo independiente como dibujantes, proyectistas o calculistas funciona como actividad «refugio», de manera que «la posibilidad de trabajar como independiente siempre está» (Leandro, ingeniero civil, julio 2010). Además, «[...] bajan las condiciones laborales, bajan las ofertas», en la medida en que no hay obras nuevas «no hay un ingeniero que trabaje, entonces se prescinde del puesto de trabajo.Y como las obras tienen [...] siempre un plazo de terminación, si no sale otra obra nueva que la reemplaza, es un puesto de trabajo que no continúa» (Facundo, ingeniero civil, agosto 2012).

La alta concentración empresaria a nivel de grandes obras, la rotación de pymes y la tercerización de subcontratistas es una característica de este sector en el cual se trabaja "por proyecto". Los procesos productivos son variables y esta situación incide en la flexibilización empresaria (Panaia, 2004). La posición subordinada que ocupan los estudios de ingeniería en estas redes de subcontratación explica las condiciones de trabajo de los ingenieros que son empleados en estos estudios con salarios que no cumplen con sus expectativas y con poca participación en la concepción del producto. Así se manifiesta un entrevistado graduado en el año 2008 que trabaja en un estudio de ingeniería haciendo cálculos estructurales para la construcción de estaciones transformadoras de energía:

\section{[...] A nosotros nos contrata una empresa, que agarra una licitación, a su vez a esa la con- trata otra empresa, esa a otra y esa otra hace una parte y la parte civil la hacemos nosotros [...] somos el último orejón del tarro... (Justo, ingeniero civil, abril 2012)}

Ante los condicionamientos del contexto económico, sectorial y del mercado profesional, aparece en algunos relatos la inquietud vocacional junto con un proyecto fuerte y claro de construcción del propio camino de inserción profesional. Ese camino es tomado como un desafio cuando comienzan la carrera en años de crisis económica o en un país que compra «llave en mano» la construcción de grandes obras de infraestructura. En los casos en que la inquietud vocacional es fuerte, el conocimiento práctico del sector demandante - que es cambiante y hostil- y la formación universitaria brindan las fortalezas para la realización personal aun si el sueño es "construir puentes», como comenta 
un entrevistado, de modo que «si no está el ámbito habrá que generarlo, si no habrá que ir a otro ámbito» (Andrés, ingeniero civil, agosto 2010).

A modo de cierre, en el próximo apartado y en base a los relatos de los graduados, se muestran la valoración social y subjetiva del rol profesional, el reconocimiento de sus capacidades y la posibilidad de ponerlas en práctica, el nivel salarial y las perspectivas profesionales.

\section{Los problemas de inserción profesional}

Como se viene señalando a lo largo del artículo, tanto los factores relacionados con los cambios de plan de carrera, de incumbencias y de régimen de estudio como los factores vinculados con el sector de la construcción y el mercado de trabajo impactan en las trayectorias educativas y laborales de los graduados y en estos casos analizados se traducen en posiciones profesionales desdibujadas, desvalorización del título e incertidumbre en cuanto a las posibilidades de su ejercicio en el mercado laboral.

Se desdibuja el rol profesional por tres vías. Una es que las tareas y puestos se alejan poco del trabajo técnico; la segunda es la falta de posicionamiento colectivo respecto de otras profesiones que compiten en el sector de la construcción, y la tercera está marcada por la incertidumbre de poner en práctica las capacidades en su nivel de competencias ya que la actividad de grandes obras es cambiante y temporal.

Cuando se interroga por la valoración de la especialidad, vuelve a surgir la vinculación con los cambios económicos.

Se valora cuando hay trabajo. Civil [...] es una especialidad que es muy variable con el tiempo con la realidad económica de la Argentina. No es algo que se mantenga. El país crece, crecen las obras. Y el país crece, por lo menos en los últimos años, a base de obras públicas, y las obras públicas tienen consecuencia en obras privadas, y la obra privada surge de un buen estado económico. Entonces cuando hay mucho trabajo sí que está bien visto, pero cuando baja un poco el trabajo, por ejemplo este último año [2012], y la expectativa que se tiene de acá para adelante, no esta tan bien valorado. (Facundo, ingeniero civil, agosto 2012)

En la mayoría de los relatos biográficos se resalta que en el ámbito laboral no está claramente definido el rol profesional, identificado para los ingenieros con tareas principalmente conceptuales, por oposición a la del técnico, que está ligada a la resolución de problemas empíricos. Si bien es cierto que contempla dimensiones técnicas, también incluye las jerárquicas y de gestión (Lanciano y Nohara, 1997). Estas últimas son difíciles de alcanzar para los graduados entrevistados, aun cuando poseen trayectorias laborales extensas y experiencia en tareas del sector luego de tres años de obtención del título de ingeniero, como los ejemplos que se citan a continuación.

[...] [mis conocimientos están aprovechados] por debajo, porque yo tengo un nivel de conocimiento que afecta varias áreas que ni siquiera estoy viendo, sí uso un $10 \%$ de los conocimientos que aprendí acá [facultad] que me sirve perfectamente para cumplir lo que me están pidiendo, y si el día de mañana avanzo a nivel jerárquico seguramente llegaré a un $20 \%$ [...] Porque tengo otras cosas que tienen que ver con la parte legal, que afectan otro tipo de decisiones, yo no tengo tanto poder de decisión, tengo $[\ldots]$ pero chiquito, entonces no se está aprovechando mi nivel de conocimientos. (Marcelo, ingeniero en cons- trucciones e ingeniero civil, septiembre 2010)

Vos estás sobrecalificado y terminás haciendo un trabajo que no llena las expectativas. (Federico, ingeniero en construcciones, abril 2012)

Se observa en algunas de las trayectorias que la obtención del título lleva a la decisión de buscar otro empleo que reconozca el nuevo estatus no solo como un mero cambio de categoría, sino como un cambio en el contenido del trabajo y/o en áreas relacionadas con la práctica de la ingeniería. Como ellos plantean, a partir del título «se generan otras situaciones» y este permite el contacto "con gente distinta» y el ofrecimiento de un trabajo extra. Sin embargo, para aquellos con trayectorias de trabajo independiente no representa un cambio significativo o de contenido del trabajo, sino solo de habilitación para construcciones de mayor envergadura. De forma que "sí con el título puedo firmar un edificio de 10 pisos que antes no [como maestro mayor de obra puede firmar hasta cuatro pisos] [...] pero no hubo gran diferencia entre lo que venía haciendo con lo de hoy» (Andrés, ingeniero en construcciones, agosto 2010).

Algunos dicen no haber realizado el trámite de matriculación en los colegios profesionales, porque otros "ponen las firma» a pesar de ser ellos quienes realizan el trabajo en su integralidad. Este procedimiento permite desempeñarse en puestos y tareas de ingeniero con la formación acorde pero sin el requisito de la obtención del título. Es de destacar que son pocos los que aluden a la necesidad de poseer el título para trabajar en los puestos del empleo actual. El caso de un empresario, segunda generación en la empresa constructora - el padre posee el título técnico de maestro mayor de obra-, aparece como una excepción, ya que hace alusión a su matrícula y a la responsabilidad de su firma en las obras civiles. A partir de su incorporación, la empresa 
se diversifica hacia un perfil de obras de infraestructura en las cuales utiliza su título habilitante de ingeniero civil. Sus tareas son más de gestión, conducción y de coordinación del negocio, pero firma y tiene la responsabilidad profesional de las obras. Coincide con que es una de las pocas trayectorias que muestran un importante nivel de satisfacción y desarrollo profesional.

En cambio, la mayoría de los casos presentan trayectorias de siete a diez empleos, movilidad que se relaciona con constantes búsquedas de mejoras salariales y puestos acordes con la profesión, además de búsquedas que se adelantan al momento de terminación de los proyectos de construcción.

Las alternativas de contratación que encuentran los ingenieros los posicionan en un lugar intermedio entre el ejercicio de su profesión en relación de dependencia y como independientes o autónomos. Es ilustrativa la experiencia que se transcribe aquí:

... y no nos pusimos de acuerdo con la remuneración, o sea, ellos querían contratarme en relación de dependencia pero no me podían pagar lo que yo pedía, entonces surgió la idea que yo haga trabajos para ellos como autónomo [...] Bueno, siempre con un arreglo del precio, ¿no?, y una vez que hice el primer trabajo, ya está el contacto, ya saben la forma que uno trabaja y después depende de uno. (Antonio, ingeniero civil, septiembre 2010)
Los niveles salariales no cubren las expectativas de los graduados en las empresas pequeñas y medianas que responden a estructuras familiares e informales. En las grandes, por su parte, al ser profesionales y estar fuera de las convenciones colectivas de trabajo, muchas veces su nivel salarial queda desfasado respecto del de los empleados de nivel técnico que sí están encuadrados en convenios. Por otra parte, el poco acceso a puestos jerárquicos atenta contra el nivel salarial de estos profesionales que relatan la falta de reconocimiento de su trabajo y su profesión en materia de ingresos. Esta situación excede el tipo de empresa que los emplea, como ilustra el siguiente relato.

[...] me gustaría que me paguen más, o sea, me gustaría seguir porque estoy cómodo, porque los proyectos son ambiciosos, son desafiantes, no hay muchas empresas en este país que encaren proyectos de esta magnitud $[\ldots]$ pero me gustaría también que me paguen más por el trabajo que estoy haciendo. (Germán, ingeniero civil, septiembre 2010)

Como se menciona anteriormente, los niveles de ingresos, casi equivalentes a los de un técnico con antigüedad, explican la movilidad laboral, el rol profesional con poco reconocimiento y el desinterés en la obtención del título en el tiempo «nominal» de los planes.

\section{Consideraciones finales}

El análisis en profundidad de las trayectorias laborales y educativas de las cohortes recientes de graduados de la carrera de Ingeniería Civil y de Construcciones de la UTN-FRA permite reflexionar sobre los problemas de interrupción, abandono y extensión de los trayectos universitarios, ampliando la óptica de los factores educativos-institucionales (cambios de plan, régimen de estudio, cambios de titulación, incumbencias) a las características del sector productivo que los demanda y en el cual se desempeñan como estudiantes avanzados y como profesionales. En este sentido, los problemas de inserción profesional y del mercado de trabajo no son ajenos a las modalidades de estudio, a la duración de las carreras y a la valoración de la titulación. Como se ha concluido en este artículo, la situación de carreras extensas y con interrupciones se relaciona con el contexto económico y el mercado profesional. Este último como un terreno en disputa en el cual la posesión del título no asegura - en estos grupos estudiados - una posición definida en el mercado de trabajo profesional. La obtención del diploma se vivencia como una meta poco valorizada en el caso de trabajadores con amplia experiencia en el rubro. El esfuerzo por la obtención de la titulación en esta carrera de ingeniería - para las generaciones de graduados analizadas - entra en tensión con los problemas de inserción profesional en el contexto económico y social en el que se desempeñan y las características de la actividad que principalmente los demanda en el país. 


\section{Referencias bibliográficas}

Arana, M. (2004). La retención de alumnos en universidad pública argentina. Análisis de la Facultad Regional Avellaneda de la Universidad Tecnológica Nacional. Ponencia en el IV Colóquio Internacional sobre Gestão Universitária na América do Sul. Disponible en http://repositorio.ufsc.br/xmlui/handle/123456789/35767.

CIPE (2012). Carreras de Ingeniería Plan Estratégico 2012-2016. Programa de Calidad Universitaria, spu, Ministerio de Educación (mimeo).

Coremberg, A. (2000). La reconversión productiva en el sector de la construcción en Argentina durante la década del 90. XXXV Reunión Anual de la AAEP. Córdoba: Ministerio de Economía (mimeo).

Dubar, C. (2001). El trabajo y las identidades profesionales y personales. Revista Latinoamericana de Estudios del Trabajo. Buenos Aires, año 7, n. ${ }^{\circ} 13,5-16$.

EnRich, H. (2014). Desempeño estudiantil en la Facultad de Ingeniería. InterCambios, vol. 2, n. ${ }^{\circ}$ 1, diciembre, 41-47.

Formento, M. C.; Weglin, J. y Freiberger, S. (2007). Las carreras de Ingeniería Mecánica e Ingeniería Civil. Los datos de cinco generaciones. Documento de Trabajo n. ${ }^{\circ}$ 6, General Pacheco: MIG Facultad General Pacheco Universidad Tecnológica Nacional.

Fuchs, M. y Vispo, A. (1995). Diagnóstico sobre la demanda futura de ingenieros. Buenos Aires: CePal.

GODARD, F. y CABANES, R. (1996). Uso de las historias de vida en ciencias sociales. Centro de Investigaciones sobre Dinámica Social, serie II, Bogotá, Universidad Externado.

Jacinto, C. (comp.) (2010). La construcción social de las trayectorias laborales de jóvenes. Buenos Aires: Teseo-IDEs.

Lanciano, C. y Nohara, H. (1997). Socialización de los ingenieros y construcción de sus competencias: comparación internacional. Documento de Trabajo n. ${ }^{\circ}$ 16. Calificaciones y Empleo, Buenos Aires: Ceil/Piette Conicet.

LANDi, J.A. y Giuliodori, R. F. (2001). Graduación y deserción en las universidades nacionales. En JozAmi, A. y SÁnchez Martínez (comps.). Estudiantes y profesionales en la Argentina. Buenos Aires: eduntref.

Longo, M. E. (2008). Claves para el análisis de las trayectorias profesionales de los jóvenes: multiplicidad de factores y de temporalidades. Estudios del Trabajo (35), 73-95.

Lujambio, V., González, S. y Santiviago, C. (2013). Experiencia Plan 80. Libro de trabajos y resúmenes del seminario internacional de intercambio de experiencias e investigaciones sobre egreso universitario. Uruguay: Facultad de Veterinaria y Facultad de Agronomía, Universidad de la República.

PAiLOT, P. (2003). Méthode biographique et entrepreneuriat: Application à l'étude de la socialization entrepreneuriale anticipée. Revue de l'Entrepreneuriat, vol. 2, n. ${ }^{\circ}$ 1, 2003, 19-41.

Panaia, M. (2004). El sector de la construcción: un proceso de industrialización inconcluso. Buenos Aires: Nobuko.

- (2006a). Trayectorias de ingenieros tecnológicos. Graduados y alumnos en el mercado de trabajo. Buenos Aires-Madrid: Miño y Dávila.

- (2006b). Una revisión de la sociología de las profesiones desde la teoría crítica del trabajo en la Argentina. Estudios del Trabajo, Buenos Aires, n. ${ }^{\circ} 32,121-165$.

- (2008). Un puente entre la universidad y el mercado de trabajo. Revista Argentina de Enseñanza de la Ingeniería, año 9 , n. ${ }^{\circ}$ 17. Córdoba: Editorial de la Fundación de la Universidad Nacional de Río Cuarto, 7-26.

- (coord.) (2011). Trayectorias de graduados y estudiantes de ingeniería. Buenos Aires: Biblos.

- (2013) Abandonar la universidad: ¿Decisión premeditada o imprevista? En Panaia, Marta (coord.) Abandonar la universidad con o sin título (21-65). Buenos Aires-Madrid: Miño y Dávila

Ruggirello, H. (2011). El sector de la construcción en perspectiva: internacionalización e impacto en el mercado de trabajo. Buenos Aires: Aulas y Andamios.

Simón, M. (2015). Ingeniería para el futuro. Revista Enlaces, año 8, n. ${ }^{\circ} 13$. Montevideo, Universidad de la República, Facultad de Ingeniería, Fundación Julio Ricaldoni. Disponible en http://www.ricaldoni.org.uy/revistaenlacesfing.

Simone,V. y Wejchenberg, D. (2013). Una visión de conjunto sobre los ingenieros graduados en los años 2006 y 2007 de la UTN-Fra. En Panaia, Marta (coord.) Abandonar la universidad con o sin título (111-138). Buenos Aires-Madrid: Miño y Dávila.

Simone,V.; Campetelli,V.; Pagotto, A. y Wejchenberg, D. (2007). Análisis institucional y estudio de las carreras de la utnFRA (Documento de Trabajo n. ${ }^{\circ}$ 1). Avellaneda: mig Facultad Regional Avellaneda Universidad Tecnológica Nacional.

Simone,V.; IAvorski, I. y Somma, L. (2013). Los ingenieros y la ingeniería. Una mirada desde la formación y la trayectoria profesional. Sitio web delVII Congreso Latinoamericano de Estudios del Trabajo «El trabajo en el siglo xxi. Cambios, impactos y perspectivas». San Pablo, Brasil, julio de 2013. Disponible en http://alast2013.com.br/gt17.asp.

Simone,V.; IAVORSKi, I. y WejChenberg, D. (2012). Formación y procesos de inserción laboral de ingenieros. Comparación entre los graduados de las seis especialidades de ingeniería de la UTN-FRA (Documento de Trabajo n. ${ }^{\circ}$ ). Avellaneda: MIg Facultad Regional Avellaneda, Universidad Tecnológica Nacional. 